\title{
Perception and Evaluation of Psychological Stress Awareness during Corona Virus Disease among General Population in Karachi, Pakistan.
}

\author{
*Akhtar Shareef ${ }^{1}$, Durdana Rais Hashmi ${ }^{2}$ and Muhammad Azam ${ }^{3}$ \\ ${ }^{1,2}$ Centre for Environmental Studies, \\ Pakistan Council of Scientific and Industrial Research Laboratories Complex, Karachi, Pakistan \\ ${ }^{3}$ Department of Geography, \\ Federal Urdu University of Arts, Sciences and Technology, Karachi, Pakistan \\ Email Address: akhtarshareef@yahoo.com,drhpakr@yahoo.com and mhdazam11@hotmail.com \\ *Corresponding author, Email Address: akhtarshareef@yahoo.com \\ DOI: 10.29322/IJSRP.11.03.2021.p11179 \\ http://dx.doi.org/10.29322/IJSRP.11.03.2021.p11179
}

\begin{abstract}
:
Background: The main object of this study was to analyze the opinion of general public in Karachi city to examine the levels of psychological impact (anxiety, stress and depression) during the $1^{\text {st }}$ episode of the COVID-19 pandemic. Coronavirus epidemic is a public health emergency of international distress and poses a challenge to psychological resilience. The collected data will be used for future reference.
\end{abstract}

Methods: An online questionnaire survey was conducted from April to May, 2020. The survey was conducted by using snowball sampling techniques, virtual learning environments and social networking (e.g. Whats-App and Facebook groups). The online collected data were based on socio-demographic characters comprised on age, gender, level of education, marital status and occupational background, physical symptoms during the past 14 days, knowledge and fear, precautionary measures and supplementary data regarding the COVID-19 infection. DASS(21) scale was used to analyze the status of mental helth of common habitants during COVID infection.

Results: In this survey 510 participants were participated from different areas of Karachi city. For stress 189(51.4\%) male and 63(44\%) female were considered to have normal score (0-10), mild to moderate (11-26) in 112 (30.5\%) male and 42(29.3\%) female, severe to extremely severe score (27-42) was found among 66(18\%) male and 38(1926.5\%) female respondents. For depression subscale, 152 (41.4\%) male and 56 (39\%) female were considered to have normal score (0-9), $110(30 \%)$ male and $42(29 \%)$ female considered mild to moderate score (10-20) and severe to extremely severe score (21-42) was found among 105(29\%) male and 45(31.4\%) female respondents. For anxiety subscale, 142(39\%) male and 58(40\%) female were considered to have normal score (07). Mild to moderate score (8-14) was of 101(28\%) male and 37 (26\%) were female. Severe to extremely severe score (15-42) was found among 124(34\%) male and $48(33 \%)$ were female participants. There is no significance of anxiety as marital status and education wise. Only the students are anxious about their studies and future.

Conclusions: High levels of anxiety, stress and depression among general population were noted, which is a cause for concern. Both the government and health care agencies are responsible for protecting the psychological well-being all over the world and ensuring a healthy work environment.

Keywords: coronavirus; epidemic; anxiety; stress; depression; respiratory symptoms; psychological impact; knowledge and precaution;

\section{Introduction}

The worldwide pandemic of noval coronavirus (COVID-19) has wrecked the physiological distress, mental health and economy of the world. In Pakistan, during first episode of the pandemic, more than 200,000 cases had been reported and 4,000 people had died. As the COVID Pandemic is spreading rapidly, it has caused panic and fear among people of all age groups of habitants. Moreover, this pandemic has also caused psychological distress due to contagious nature, absence of vaccine, treatment facilities, and social distancing that has been forced upon every individual, which may resulted to feeling of loneliness and helplessness. The widespread 
news coverage on media, Facebook, YouTube, regarding the pandemic has caused dread of the COVID symptoms, fear, stress, anxiety, and depression and also uncertainty in the environment [1,2].

In China during the research on psychological responses and associated factors among the general population during COVID-19 epidemic estimate that $53.8 \%$ of participants reported stress, anxiety, and depression [2]. More-over, result of the study also stated that three-quarters of the participants were anxious about their family due to this pandemic [2]. Similarly, in Pakistan, the outbreak of pandemic (COVID-19) has also caused Depression, Anxiety and stress in people [1].

Common symptoms of 2019 infection are fever, chills, cough, coryza, sore throat, breathing difficulty, myalgia, nausea, vomiting, and diarrhea found in the infected person [3], whereas severe symptoms are cardiac injury, respiratory failure, acute respiratory distress syndrome, and death [4]. Aged citizens are at high risk due to medical comorbidities [3]. According to WHO report the interim mortality rate in $1^{\text {st }}$ episode of COVID is around $2 \%$, whereas other researchers estimate $0.3 \%$ to $0.6 \%$ mortality rate [5]. Social media platforms, such as Facebook, You tube, and several others are also being extensively used to promote healthy lifestyles and the adoption of coping mechanisms during the lockdown period. Moreover, to minimize the negative impact of the lockdown, online modalities and virtual modes of engaging in education, work, and socialization with friends, and families are being regularly conveyed to the people. These platforms are also constantly providing various activities that people can undertake for a healthy lifestyle, like sports, yoga, music, arts, and mindfulness. Moreover, governmental agencies have made efforts to ensure the availability of masks, hand sanitizers, and soaps; particularly at religious places, business centers, shops, workplaces, and on public transport, to reduce the fear and anxiety associated with contracting the infection, among people.

Aim of this survey is to establish the prevalence of psychiatric symptoms, categorized the risk factor and protective measures to minimize the psychological stress during the face of COVID-19. Survey also helps government agencies, doctors, clinical staffs, front line workers and well-wishers of community to subsidize the expansion of outbreak in all the cities of the country.

\section{Method}

A web-based questionnaire survey was projected to evaluate the status of psychological status of the citizens in the COVID-19 situation. The Questionnaire was developed, after endorsement from three experts, who were working as scientist and senior consultants in research organization with more than 15 year experience. This cross-sectional survey was conducted from April to May, 2020. An open-ended questionnaire was developed, asking the participants about the challenges associated with personal and professional impact related to COVID-19 pandemic. The population under study consisted of general population including students, shopkeepers, office workers, teachers, business man and those who were willing to participate in this study. We prepared questionnaire consisting of DASS-21 tool and also the questionnaire related to Socio-demographic factors. Socio-demographic data were comprised on age, gender, level of education, marital status and occupational background.

The questionnaire was shared through email, snowballing, virtual learning environments and social networking (e.g.Whats-App and Facebook groups). Data collection took place over ten to fifteen days (20 ${ }^{\text {th }}$ April to $7^{\text {th }}$ May, 2020). The sample size was not encoded, data collection and analysis was taken until data and time saturation were reached. Sample size was calculated by using open Epi calculator. All the respondents were provided informed consent.

Measurement / monitoring regarding the mental health status was carried out by using the DASS-21 tool scale, to calculate the responsive states of depression, anxiety, stress scale [6].

\subsection{Developmental Survey}

Few Questions designed for the depression subscale. The depression subscale was divided into 5 modes as normal (0-9), mild depression (10-13), moderate depression (14-20), severe depression (21-27), and extremely severe depression (28-42). Another few questions designed for the anxiety subscale. The anxiety subscale was also divided into normal $(0-7)$, mild anxiety $(8-9)$, moderate anxiety (10-14), severe anxiety (15-19) and extremely severe anxiety (20-42). Similarly few Questions designed for the stress subscale and was divided into the same 5 modes as normal $(0-14)$, mild stress (15-18), moderate stress (19-25), severe stress (2633), and extremely severe stress (34-42) presented in Table-1. The DASS has been demonstrated to be a reliable and valid measure in assessing mental health [7,8]. The DASS scale was also used during the SARS pandemic [9].

\begin{tabular}{||l|c|c|c||}
\hline \multicolumn{1}{|c|}{ Meaning } & Depression & Anxiety & Stress \\
\hline \hline Normal & $0-9$ & $0-7$ & $0-14$ \\
\hline Mild & $10-13$ & $8-9$ & $15-18$ \\
\hline Moderate & $14-20$ & $10-14$ & $19-25$ \\
\hline Severe & $21-27$ & $15-19$ & $26-33$ \\
\hline Extremely severe & $28+$ & $20+$ & $34+$ \\
\hline
\end{tabular}

This publication is licensed under Creative Commons Attribution CC BY. 
Source:- Lovibond SH, Lovibond PF:Manual for the Depression Anxiety Stress Scales.

2nd edition. Sydney: Psychology Foundation of Australia; 1995.[6].

\subsection{Survey Respondents}

An online questionnaire survey was conducted to evaluate the public's psychological effects and awareness during the COVID-19 pandemic regarding the health status according to DASS-21 scale (Depression, Anxiety and Stress Scale) to calculate the situations of depression, anxiety and stress during corona virus disease in general habitant of Karachi city.

\subsection{Sociodemographic Variables and Psychological Impact.}

Socio-demographic data were collected on the basis of gender, age, education, marital status and occupation. Participants response the status of symptoms found during the infection of COVID and feel fever, chills, headache, myalgia, cough, difficulty in breathing, dizziness, coryza, sore throat, persistent fever and difficulty in breathing. Participants of this survey were also presented the data about the frequency of their mental health status and history of chronic ailment. Questionnaire survey also contains a section of health examination i-e consultation with area doctor, visit to nearest clinic, admission to the hospital, being quarantined by a health authority, and being tested for COVID-19.

Knowledge about COVID-19 variables, such as routes of transmission, fear from increasing the infection, satisfaction of available health information about this infection, inclination of new cases, treatment and mortality rate due to infection. In the prescribed questionnaire participants were also asked to specify source of information, worries about COVID infection for himself, for family members and worries about the chance of surviving in case of infected by COVID virus.

\section{Results}

\subsection{Development of the COVID-19 Epidemic from April to May 2020.}

Figure 1 presented the developmental trend of corona virus disease in Karachi city from April to May, 2020. The first case of coronavirus was reported from Karachi on February 26, 2020. Since then the number of confirmed, suspected, recovered cases and mortality due to corona infection continuously become worsen day by day. Children and Aged citizens are more vulnerable to this virus.

\subsection{Sociodemographic Variables and Psychological Impact}

Table -1 shows that total 510 participants interviewed in this online questionnaire study. All of the participants were above 18 years of age divide into three categories as between 16-29 years (47.5\%), 30-44 years (28\%) and 24.5\% were between 45 to 60 years of age. Study reveals that approximately $72 \%$ of the respondents were male and $28 \%$ were Female in gender. Participants included in this study were those who have access to internet facility. Lowest level of education was observed in this study is up to $9^{\text {th }}$ standard. The uppermost qualification of the participants $62 \%$ were graduation and above. Out of them most were those having Private jobs and office worker in his profession. Table-1 also show the percentage of the respondent participants according to the level of education, $18 \%$ of the respondents were educated up to secondary school certificate (SSC) and from the rest of 82\% respondents, 20\% were educated up to higher secondary school certificate (HSC) whereas 62\% having graduate and above level of education. \% of respondents according to the occupation and marital status are also presented in table-1 respectively. Surprisingly, most of the participants $49.2 \%$ were students in this study, who were very anxious about their future. COVID-19 pandemic have significant adverse impact on the mental health of Pakistani university / college students; prevalence of moderate anxiety was $43.8 \%$ whereas $50.0 \%$ students were with severe depression.

Table 1. Demographic Characters and Association between physical health status among general population during COVID19 Pandemic $(\mathbf{n}=510)$.

\begin{tabular}{|c|c|c|c|c|c|c|c|c|c|c|c|}
\hline \multirow{2}{*}{\multicolumn{2}{|c|}{ Variable }} & \multirow{3}{*}{$\begin{array}{c}\mathbf{N}(\%) \\
242 \\
(47.5)\end{array}$} & \multicolumn{3}{|c|}{ Stress in $\mathbf{n}(\%)$} & \multicolumn{3}{|c|}{ Depression in $\mathbf{n}(\%)$} & \multicolumn{3}{|c|}{ Anxiety in $n(\%)$} \\
\hline & & & Normal & Moderate & Severe & Normal & Moderate & Severe & Normal & Moderate & Severe \\
\hline \multirow{3}{*}{ 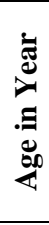 } & $16-29$ & & $\begin{array}{c}112 \\
(46.3 \%) \\
\end{array}$ & $\begin{array}{c}86 \\
(35.5 \%) \\
\end{array}$ & $\begin{array}{c}44 \\
(18.2 \%) \\
\end{array}$ & $\begin{array}{c}126 \\
(52.1 \%) \\
\end{array}$ & $\begin{array}{c}66 \\
(27.3 \%) \\
\end{array}$ & $\begin{array}{c}50 \\
(20.1 \%) \\
\end{array}$ & $\begin{array}{c}116 \\
(48 \%) \\
\end{array}$ & $\begin{array}{c}52 \\
(22 \%) \\
\end{array}$ & $\begin{array}{c}74 \\
(30.6) \\
\end{array}$ \\
\hline & $30-44$ & $\begin{array}{c}143 \\
(28.0) \\
\end{array}$ & $\begin{array}{c}70 \\
(49 \%) \\
\end{array}$ & $\begin{array}{c}60 \\
(42 \%) \\
\end{array}$ & $\begin{array}{c}13 \\
(9.1 \% \\
\end{array}$ & $\begin{array}{c}77 \\
(54 \%) \\
\end{array}$ & $\begin{array}{c}56 \\
(39.2 \%) \\
\end{array}$ & $\begin{array}{c}10 \\
(7 \%) \\
\end{array}$ & $\begin{array}{c}90 \\
(63 \%) \\
\end{array}$ & $\begin{array}{c}33 \\
(23.1 \%) \\
\end{array}$ & $\begin{array}{c}20 \\
(14 \%)\end{array}$ \\
\hline & $45-60$ & $\begin{array}{c}125 \\
(24.5) \\
\end{array}$ & $\begin{array}{c}15 \\
(12 \%) \\
\end{array}$ & $\begin{array}{c}49 \\
(39 \%) \\
\end{array}$ & $\begin{array}{c}61 \\
(49 \%) \\
\end{array}$ & $\begin{array}{c}10 \\
(8 \%)\end{array}$ & $\begin{array}{c}33 \\
(26.4 \%) \\
\end{array}$ & $\begin{array}{c}82 \\
(65.6 \%) \\
\end{array}$ & $\begin{array}{c}25 \\
(20 \%) \\
\end{array}$ & $\begin{array}{c}10 \\
(8 \%) \\
\end{array}$ & $\begin{array}{c}90 \\
(72 \%) \\
\end{array}$ \\
\hline \multirow{2}{*}{ ن⿺辶ِّ } & Male & $\begin{array}{c}367 \\
(72 \%)\end{array}$ & $\begin{array}{c}189 \\
(51.4 \%) \\
\end{array}$ & $\begin{array}{c}112 \\
(30.5)\end{array}$ & $\begin{array}{c}66 \\
(18.0 \%) \\
\end{array}$ & $\begin{array}{c}152 \\
(41.4 \%) \\
\end{array}$ & $\begin{array}{c}110 \\
(30 \%) \\
\end{array}$ & $\begin{array}{c}105 \\
(28.6 \%) \\
\end{array}$ & $\begin{array}{c}142 \\
(38.7 \%) \\
\end{array}$ & $\begin{array}{c}101 \\
(27.5 \%) \\
\end{array}$ & $\begin{array}{c}124 \\
(33.7 \%) \\
\end{array}$ \\
\hline & Female & $\begin{array}{c}143 \\
(28 \%)\end{array}$ & $\begin{array}{c}63 \\
(44 \%)\end{array}$ & $\begin{array}{c}42 \\
(29.3 \%)\end{array}$ & $\begin{array}{c}38 \\
(26.5 \%)\end{array}$ & $\begin{array}{c}56 \\
(39.1 \%)\end{array}$ & $\begin{array}{c}42 \\
(29.3 \%)\end{array}$ & $\begin{array}{c}45 \\
(31.4 \%)\end{array}$ & $\begin{array}{c}58 \\
(40.5 \%)\end{array}$ & $\begin{array}{c}37 \\
(26 \%)\end{array}$ & $\begin{array}{c}48 \\
(33.5 \%)\end{array}$ \\
\hline
\end{tabular}

This publication is licensed under Creative Commons Attribution CC BY. 


\begin{tabular}{|c|c|c|c|c|c|c|c|c|c|c|c|}
\hline \multirow{3}{*}{ 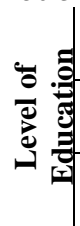 } & Up to $S S C$ & $\begin{array}{c}90 \\
(18 \%)\end{array}$ & $\begin{array}{c}35 \\
(39 \%)\end{array}$ & $\begin{array}{c}30 \\
(33.3 \%)\end{array}$ & $\begin{array}{c}25 \\
(28 \%)\end{array}$ & $\begin{array}{c}47 \\
(52.2 \%)\end{array}$ & $\begin{array}{c}33 \\
(36.6 \%)\end{array}$ & $\begin{array}{c}10 \\
(11.1 \%)\end{array}$ & $\begin{array}{c}43 \\
(48 \%)\end{array}$ & $\begin{array}{c}35 \\
(39 \%)\end{array}$ & $\begin{array}{c}12 \\
(13.3 \%)\end{array}$ \\
\hline & HSC & $\begin{array}{c}100 \\
(20 \%)\end{array}$ & $\begin{array}{c}50 \\
(50.0 \%)\end{array}$ & $\begin{array}{c}30 \\
(30.0 \%)\end{array}$ & $\begin{array}{c}20 \\
(20.0 \%)\end{array}$ & $\begin{array}{c}50 \\
(50 \%)\end{array}$ & $\begin{array}{c}20 \\
(20 \%)\end{array}$ & $\begin{array}{c}30 \\
(30 \%)\end{array}$ & $\begin{array}{c}46 \\
(46 \%)\end{array}$ & $\begin{array}{c}31 \\
(31 \%)\end{array}$ & $\begin{array}{c}23 \\
(23 \%)\end{array}$ \\
\hline & $\begin{array}{r}\text { Graduate } \\
\text { \& above }\end{array}$ & $\begin{array}{c}320 \\
(62 \%)\end{array}$ & $\begin{array}{c}183 \\
(57.2 \%)\end{array}$ & $\begin{array}{c}73 \\
(23 \%)\end{array}$ & $\begin{array}{c}64 \\
(20 \%)\end{array}$ & $\begin{array}{c}210 \\
(65.6 \%)\end{array}$ & $\begin{array}{c}74 \\
(23 \%)\end{array}$ & $\begin{array}{c}36 \\
(11.2 \%)\end{array}$ & $\begin{array}{c}172 \\
(54 \%)\end{array}$ & $\begin{array}{c}98 \\
(31 \%)\end{array}$ & $\begin{array}{c}50 \\
(16 \%)\end{array}$ \\
\hline \multirow{4}{*}{ 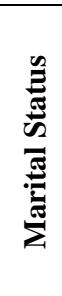 } & married & $\begin{array}{c}256 \\
(50.1 \%) \\
\end{array}$ & $\begin{array}{c}114 \\
(44.5 \%) \\
\end{array}$ & $\begin{array}{c}107 \\
(42 \%) \\
\end{array}$ & $\begin{array}{c}35 \\
(14 \%)\end{array}$ & $\begin{array}{c}71 \\
(28 \%) \\
\end{array}$ & $\begin{array}{c}127 \\
(49.5 \%) \\
\end{array}$ & $\begin{array}{c}58 \\
(23 \%) \\
\end{array}$ & $\begin{array}{c}90 \\
(35 \%) \\
\end{array}$ & $\begin{array}{c}120 \\
(47 \%)\end{array}$ & $\begin{array}{c}46 \\
(18 \%)\end{array}$ \\
\hline & $\begin{array}{r}\text { un- } \\
\text { married }\end{array}$ & $\begin{array}{c}214 \\
(42 \%) \\
\end{array}$ & $\begin{array}{c}97 \\
(43.3 \%) \\
\end{array}$ & $\begin{array}{c}90 \\
(42 \%) \\
\end{array}$ & $\begin{array}{c}27 \\
(12 \%) \\
\end{array}$ & $\begin{array}{c}70 \\
(33 \%) \\
\end{array}$ & $\begin{array}{c}106 \\
(49.5 \%) \\
\end{array}$ & $\begin{array}{c}38 \\
(18 \%) \\
\end{array}$ & $\begin{array}{c}66 \\
(31 \%) \\
\end{array}$ & $\begin{array}{c}104 \\
(49 \%) \\
\end{array}$ & $\begin{array}{c}44 \\
(20.5 \%) \\
\end{array}$ \\
\hline & divorced & $\begin{array}{c}25 \\
(5 \%)\end{array}$ & $\begin{array}{c}8 \\
(32 \%)\end{array}$ & $\begin{array}{c}12 \\
(48 \%)\end{array}$ & $\begin{array}{c}5 \\
(20 \%)\end{array}$ & $\begin{array}{c}9 \\
(36 \%)\end{array}$ & $\begin{array}{c}6 \\
(24 \%)\end{array}$ & $\begin{array}{c}10 \\
(40 \%)\end{array}$ & $\begin{array}{c}7 \\
(28 \%)\end{array}$ & $\begin{array}{c}13 \\
(52 \%)\end{array}$ & $\begin{array}{c}5 \\
(20 \%)\end{array}$ \\
\hline & widow & $\begin{array}{c}15 \\
(3 \%)\end{array}$ & $\begin{array}{c}6 \\
(40 \%)\end{array}$ & $\begin{array}{c}6 \\
(40 \%)\end{array}$ & $\begin{array}{c}3 \\
(20 \%)\end{array}$ & $\begin{array}{c}5 \\
(33.3 \%)\end{array}$ & $\begin{array}{c}9 \\
(60 \%)\end{array}$ & $\begin{array}{c}1 \\
(6.6 \%)\end{array}$ & $\begin{array}{c}3 \\
(20 \%)\end{array}$ & $\begin{array}{c}3 \\
(20 \%)\end{array}$ & $\begin{array}{c}9 \\
(60 \%)\end{array}$ \\
\hline \multirow{4}{*}{ 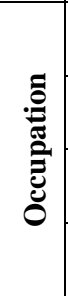 } & Student & $\begin{array}{c}251 \\
(49.2 \%)\end{array}$ & $\begin{array}{c}103 \\
(41.0 \%)\end{array}$ & $\begin{array}{c}53 \\
(21.1 \%)\end{array}$ & $\begin{array}{c}95 \\
(38.0 \%)\end{array}$ & $\begin{array}{c}66 \\
(26.6 \%)\end{array}$ & $\begin{array}{c}60 \\
(24.0 \%)\end{array}$ & $\begin{array}{c}125 \\
(50.0 \%)\end{array}$ & $\begin{array}{c}65 \\
(25.8 \%)\end{array}$ & $\begin{array}{c}110 \\
(43.8 \%)\end{array}$ & $\begin{array}{c}76 \\
(30.3 \%)\end{array}$ \\
\hline & Business & $\begin{array}{c}62 \\
(12.1 \%)\end{array}$ & $\begin{array}{c}22 \\
(35.5 \%)\end{array}$ & $\begin{array}{c}20 \\
(32.2 \%)\end{array}$ & $\begin{array}{c}20 \\
(32.2 \%)\end{array}$ & $\begin{array}{c}10 \\
(16.1 \%)\end{array}$ & $\begin{array}{c}21 \\
(34.0 \%)\end{array}$ & $\begin{array}{c}31 \\
(50.0 \%)\end{array}$ & $\begin{array}{c}25 \\
(40.3 \%)\end{array}$ & $\begin{array}{c}22 \\
(35.5 \%)\end{array}$ & $\begin{array}{c}15 \\
(24.2 \%)\end{array}$ \\
\hline & $\begin{array}{l}\text { Home } \\
\text { Maker }\end{array}$ & $\begin{array}{c}54 \\
(10.5 \%)\end{array}$ & $\begin{array}{c}22 \\
(40.7 \%)\end{array}$ & $\begin{array}{c}12 \\
(22.2 \%)\end{array}$ & $\begin{array}{c}20 \\
(37.0 \%)\end{array}$ & $\begin{array}{c}20 \\
(37.0 \%)\end{array}$ & $\begin{array}{c}20 \\
(37.0 \%)\end{array}$ & $\begin{array}{c}14 \\
(26.0 \%)\end{array}$ & $\begin{array}{c}22 \\
(41 \%)\end{array}$ & $\begin{array}{c}20 \\
(37 \%)\end{array}$ & $\begin{array}{c}12 \\
(22.2 \%)\end{array}$ \\
\hline & Jobs & $\begin{array}{c}143 \\
(28.0 \%)\end{array}$ & $\begin{array}{c}23 \\
(16.0 \%)\end{array}$ & $\begin{array}{c}40 \\
(28.0 \%)\end{array}$ & $\begin{array}{c}80 \\
(56.0 \%)\end{array}$ & $\begin{array}{c}30 \\
(21 \%)\end{array}$ & $\begin{array}{c}43 \\
(30 \%)\end{array}$ & $\begin{array}{c}70 \\
(49 \%)\end{array}$ & $\begin{array}{c}43 \\
(30 \%)\end{array}$ & $\begin{array}{c}53 \\
(37 \%)\end{array}$ & $\begin{array}{c}47 \\
(33 \%)\end{array}$ \\
\hline
\end{tabular}

\subsection{Symptoms and Psychological Impact}

Table 2 shows physical symptoms and impact due to Virus. Table 2 presented that $1.0 \%$ of the participants testified fever of $38^{\circ} \mathrm{C}$ for at least one day within the previous 14 days. Some of the participants illustrate a range of physical symptoms, commonly headache (17\%), myalgia (14.3\%), cough (13.3\%), coryza (13.0\%), sore throat (15.5\%), chills $(6.0 \%)$, dizziness $(7.0 \%)$ and breathing difficulty (2.0\%). Around $8.0 \%$ of participants reported a dyad of symptoms for example fever with cough $(2.0 \%)$ or fever with breathing difficulty (6.0\%), whereas only $16.0 \%$ of respondents suffer from any chronic illness. Results of the survey presented that symptoms of infection i-e chills, myalgia, cough, dizziness, coryza, and sore throat all were found in higher range of DASS stress, anxiety and depression subscale, whereas breathing difficulty was higher with only anxiety scores. Results regarding the dyad symptoms of infection (fever with cough and fever with breathing difficulty) were not found in the range of DASS scores.

Table 2. Association between physical health status and the psychological impact of COVID-19 infection and adverse mental health status during the epidemic $(n=510)$.

\begin{tabular}{|c|c|c|c|c|c|c|c|c|c|c|}
\hline \multirow[b]{2}{*}{ Variables } & \multirow[b]{2}{*}{$\mathbf{N}(\%)$} & \multicolumn{3}{|c|}{ Stress in $(\%)$} & \multicolumn{3}{|c|}{ Depression in $(\%)$} & \multicolumn{3}{|c|}{ Anxiety in (\%) } \\
\hline & & $\begin{array}{l}\overline{\text { J }} \\
\text { Z }\end{array}$ & 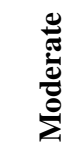 & 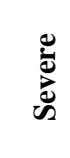 & 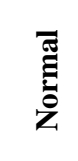 & 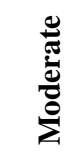 & 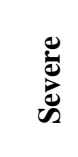 & 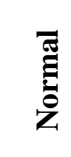 & 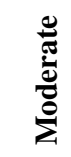 & 离 \\
\hline \multicolumn{11}{|c|}{ Persistent fever $\left(>38^{\circ} \mathrm{C}\right.$ for at least 1 day $)$} \\
\hline Yes & $5(1.0)$ & 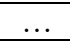 & 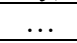 & $\ldots$ & $\ldots$ & $\ldots$ & $\ldots$ & $\ldots$ & $\ldots$ & $\ldots$ \\
\hline No & $505(99.0)$ & & & & & & & & & \\
\hline \multicolumn{11}{|l|}{ Chills } \\
\hline Yes & $31(6.0)$ & 38.2 & 14.7 & 47.1 & 34.4 & 18.2 & 48.4 & 30.2 & 19.4 & 50.4 \\
\hline No & $479(94.0)$ & & & & & & & & & \\
\hline \multicolumn{11}{|l|}{ Headache } \\
\hline Yes & $87(17.0)$ & 34.2 & 20.5 & 45.3 & 27.2 & 26.7 & 46.2 & 26.2 & 25.5 & 48.3 \\
\hline No & $423(83.0)$ & & & & & & & & & \\
\hline \multicolumn{11}{|l|}{ Myalgia } \\
\hline Yes & $73(14.3)$ & 30.1 & 25.1 & 44.8 & 27.2 & 25.7 & 47.1 & 25.2 & 25.5 & 49.0 \\
\hline No & $437(85.6)$ & & & & & & & & & \\
\hline \multicolumn{11}{|l|}{ Cough } \\
\hline Yes & $68(13.3)$ & 28.0 & 27.0 & 45.0 & 27.0 & 25.0 & 48.0 & 27.0 & 26.0 & 47.0 \\
\hline No & $442(86.6)$ & & & & & & & & & \\
\hline
\end{tabular}

This publication is licensed under Creative Commons Attribution CC BY. 


\begin{tabular}{|c|c|c|c|c|c|c|c|c|c|c|}
\hline Yes & $10(2.0)$ & 46.0 & 30.0 & 24.0 & 41.0 & 23.0 & 36.0 & 24.0 & 26.0 & 50.0 \\
\hline No & $500(98.0)$ & & & & & & & & & \\
\hline \multicolumn{11}{|c|}{ Dizziness } \\
\hline Yes & $36(7.0 \%)$ & 35.2 & 23.5 & 41.3 & 36.0 & 23.0 & 41.0 & 35.0 & 21.0 & 44.0 \\
\hline No & $474(93.0)$ & & & & & & & & & \\
\hline \multicolumn{11}{|l|}{ Coryza } \\
\hline Yes & $65(13.0)$ & 34.0 & 21.6 & 44.4 & 29.0 & 28.0 & 43.0 & 30.0 & 23.0 & 47.0 \\
\hline No & $445(87.0)$ & & & & & & & & & \\
\hline \multicolumn{11}{|c|}{ Sore throat } \\
\hline Yes & $79(15.5)$ & 28.0 & 26.0 & 46.0 & 35.0 & 20.2 & 44.8 & 27.0 & 24.0 & 49.0 \\
\hline No & $431(84.5)$ & & & & & & & & & \\
\hline \multicolumn{11}{|c|}{ Chronic illness } \\
\hline Yes & $82(16.0)$ & 29.0 & 28.2 & 42.8 & 29.0 & 25.0 & 46.0 & 28.0 & 24.0 & 48.0 \\
\hline No & $428(84.0)$ & & & & & & & & & \\
\hline \multicolumn{11}{|c|}{ Persistent fever and cough } \\
\hline Yes & $9(2.0)$ & $\ldots$ & $\ldots$ & $\ldots$ & $\ldots$ & $\ldots$ & $\ldots$ & $\ldots$ & $\ldots$ & $\ldots$ \\
\hline No & $501(98.0)$ & & & & & & & & & \\
\hline \multicolumn{11}{|c|}{ Persistent fever and difficulty in breathing } \\
\hline Yes & $29(6.0)$ & $\ldots$ & $\ldots$ & $\ldots$ & $\ldots$ & $\ldots$ & $\ldots$ & $\ldots$ & $\ldots$ & $\ldots$ \\
\hline No & $481(94.0)$ & & & & & & & & & \\
\hline \multicolumn{11}{|c|}{ Consultation with area doctor } \\
\hline Yes & $27(5.3)$ & 30.0 & 38.0 & 32.0 & 34.0 & 30.0 & 36.0 & 17.6 & 24.2 & 53.2 \\
\hline No & $483(94.7)$ & & & & & & & & & \\
\hline \multicolumn{11}{|c|}{ Hospitalization during epidemic } \\
\hline Yes & $45(8.8)$ & 24.0 & 36.0 & 40.0 & 22.0 & 32.0 & 46.0 & 18.2 & 26.2 & 54.6 \\
\hline No & $465(91.2)$ & & & & & & & & & \\
\hline \multicolumn{11}{|c|}{ Testing for COVID-19 } \\
\hline Yes & $19(3.7)$ & 36.0 & 29.0 & 35.0 & 33.0 & 26.0 & 41.0 & 20.0 & 20.0 & 60.0 \\
\hline No & $491(96.2)$ & & & & & & & & & \\
\hline \multicolumn{11}{|c|}{ Quarantine during pandemic } \\
\hline Yes & $50(9.8)$ & 32.0 & 30.0 & 38.0 & 30.0 & 26.0 & 44.0 & 26.0 & 22.0 & 52.0 \\
\hline No & $460(90.2)$ & & & & & & & & & \\
\hline
\end{tabular}

\subsection{Health Status and Psychological Impact}

Table 2 also presented Health Status of the participants in the preceding 14 days, 5.3\% of the participants visited area doctor and nearest clinic; $8.8 \%$ hospitalize; $3.7 \%$ tested for COVID infection; $9.8 \%$ had been under self-quarantine or by the advice of doctor during infection. $\%$ of respondents regarding the consultations with area doctor and hospitalizations shows anxiety score, whereas results of chronic illness show both higher anxiety and depression scores.

\subsection{Knowledge and Fear about Spreading the COVID-19 and Psychological Impact}

Table-3 presented the results reported by the participants with respect to the knowledge about COVID-19 and presented that the most common deceptive way of virus transmission was through droplets $(85.3 \%)$, followed by contaminated objects (76.3\%), and airborne transmission $(53.1 \%)$. Fears about spreading the corona virus infection also presented in the table $-3,81.4 \%$ participants reported fear about increasing the duration of COVID-19, 80.2\% participant reported the fear regarding the health alarm about COVID-19 infection with him-self and their family members, about $85.5 \%$ of participants were worried about increasing number of COVID -19 patients, $88.4 \%$ worried about increasing number of death due to this infection whereas about $90.4 \%$ of the respondents believed that that they would be recovered / survived from COVID-19 if infected.

\subsection{Knowledge about Key Source of Health information about COVID-19 and Psychological Impact}

Table-3 also presented the report of respondents regarding the key source of health information received about COVID-19, the most common source was from the Internet (48.2\%), from different channels of TV (20.4\%) and from Radio Channels (12.0\%). The majority of the participants $(75.5 \%)$ were pleased to the government and private channels for providing the amount of health information from different sources.

Table-3 . Association between knowledge of Spreading the Virus and Source of information about COVID-19 infection during the epidemic $(n=510)$

\begin{tabular}{|c|c|c|c|}
\hline \multicolumn{4}{|c|}{ Knowledge of Spreading the infection of COVID-19 } \\
\hline Variables & $\mathbf{n}(\%)$ & Variables & $\mathbf{n}(\%)$ \\
\hline
\end{tabular}

This publication is licensed under Creative Commons Attribution CC BY. 


\begin{tabular}{|c|c|c|c|c|c|}
\hline 1- & \multicolumn{2}{|l|}{ Route of transmission } & \multirow[t]{3}{*}{ 4- } & \multicolumn{2}{|c|}{$\begin{array}{l}\text { Fear from increasing number of } \\
\text { COVID-19 Patients }\end{array}$} \\
\hline \multirow[t]{4}{*}{ a) } & \multicolumn{2}{|l|}{ Droplets } & & Yes & $436(85.5 \%)$ \\
\hline & Agree & $435(85.3 \%)$ & & No & $74(14.5 \%)$ \\
\hline & Disagree & $43(8.4 \%)$ & \multirow[t]{3}{*}{ 5- } & \multicolumn{2}{|c|}{$\begin{array}{l}\text { Fear from increasing number of } \\
\text { COVID-19 deaths }\end{array}$} \\
\hline & Do not know & $32(6.3 \%)$ & & Yes & $451(88.4 \%)$ \\
\hline \multirow[t]{4}{*}{ b) } & \multicolumn{2}{|c|}{ Contact via contaminated objects } & & No & $59(11.6 \%)$ \\
\hline & Agree & $389(76.3 \%)$ & \multirow[t]{4}{*}{ 6- } & \multirow{2}{*}{\multicolumn{2}{|c|}{$\begin{array}{l}\text { Increasing number of Recovery from } \\
\text { COVID-19 infection }\end{array}$}} \\
\hline & Disagree & $104(20.4 \%)$ & & & \\
\hline & Do not know & $17(3.3 \%)$ & & Yes & $462(90.6 \%)$ \\
\hline \multirow[t]{4}{*}{ c) } & Airborne & & & No & $48(9.4 \%)$ \\
\hline & Agree & $271(53.1 \%)$ & \multirow[t]{6}{*}{$7-$} & \multirow{2}{*}{\multicolumn{2}{|c|}{$\begin{array}{l}\text { Key source of health information about } \\
\text { COVID-19 }\end{array}$}} \\
\hline & Disagree & $135(26.5 \%)$ & & & \\
\hline & Do not know & $104(20.4 \%)$ & & Internet & $246(48.2 \%)$ \\
\hline \multirow[t]{3}{*}{ 2- } & \multicolumn{2}{|c|}{$\begin{array}{l}\text { Fear from increasing number of } \\
\text { COVID-19 duration. }\end{array}$} & & Television & $104(20.4 \%)$ \\
\hline & Yes & $415(81.4 \%)$ & & Radio & $60(12.0 \%)$ \\
\hline & No & $95(18.6 \%)$ & & Family members & $100(20.0 \%)$ \\
\hline \multirow[t]{4}{*}{ 3- } & $\begin{array}{l}\text { Fears about himself } \\
\text { members getting COVII }\end{array}$ & $\begin{array}{l}\text { and family } \\
19 .\end{array}$ & \multirow[t]{4}{*}{ 8- } & \multicolumn{2}{|c|}{$\begin{array}{l}\text { Satisfaction with the available health } \\
\text { information about COVID-19 }\end{array}$} \\
\hline & Worried for Family & $268(52.5 \%)$ & & Satisfied & $385(75.5 \%)$ \\
\hline & Worried for himself & $142(28.0 \%)$ & & Not satisfied & $100(20.0 \%)$ \\
\hline & Not worried & $100(20.0 \%)$ & & Do not know & $25(5.0 \%)$ \\
\hline
\end{tabular}

\subsection{Precautionary Measures and Psychological Impact}

Table-4 presented the Report of the participants received regarding the precautionary measures adopted by the participants during 14 days, results of the report show that (68.6\%) participants habitually washing hands after coming from outside, (63.0\%) after using the outdoor items, $(58.0 \%)$ always using a mask in the presence or absence of virus infection / symptoms, (50.5\%) participants were already well mannered and always covering mouth before coughing and sneezing and (45.1\%) always avoiding the sharing utensils (e.g., spoon, fork, glass and tea cups) during the meal.

Table-4. Relation between precautionary measures and the psychological impact of COVID-19 outbreak as well as adverse mental health status during the epidemic $(n=510)$.

\begin{tabular}{|c|c|c|}
\hline & Variables & n (\%) \\
\hline \multirow[t]{4}{*}{ 1- } & \multicolumn{2}{|c|}{ Washing hands after coming outside / Work place } \\
\hline & $\begin{array}{ll}\text { Always } \\
\end{array}$ & $350(68.6 \%)$ \\
\hline & Most of the time & $110(21.5 \%)$ \\
\hline & Sometime & $50(10.0 \%)$ \\
\hline \multirow[t]{4}{*}{ 2- } & \multicolumn{2}{|c|}{ Washing hands after touching contaminated objects } \\
\hline & Always & $320(63.0 \%)$ \\
\hline & Most of the time & $130(25.5 \%)$ \\
\hline & Sometime & $60(11.8 \%)$ \\
\hline \multirow[t]{4}{*}{ 3- } & \multicolumn{2}{|c|}{ Wearing mask in the presence or absence of symptom } \\
\hline & Always & $296(58.0 \%)$ \\
\hline & Most of the time & $84(16.5 \%)$ \\
\hline & Sometime & $130(25.5 \%)$ \\
\hline \multirow[t]{4}{*}{ 4- } & \multicolumn{2}{|c|}{ Covering mouth when coughing and sneezing } \\
\hline & Always & $258(50.5 \%)$ \\
\hline & Most of the time & $120(23.5 \%)$ \\
\hline & Sometime & $132(25.8)$ \\
\hline \multirow[t]{2}{*}{ 5- } & \multicolumn{2}{|c|}{$\begin{array}{l}\text { Avoiding sharing of utensils } \\
\text { (e.g., spoon, fork, glass and tea cups) during meals }\end{array}$} \\
\hline & Always & $230(45.1 \%)$ \\
\hline
\end{tabular}




\begin{tabular}{|r|r|r|}
\hline & Most of the time & $180(35.3 \%)$ \\
\cline { 2 - 3 } & Sometime & $100(19.6 \%)$ \\
\hline
\end{tabular}

\section{Discussion}

Result of the study shows that there was no gender wise significant difference except stress. As Stress is a feeling of emotional or physical tension. It can come from any event or thought that makes you feel frustrated, angry, or nervous. Stress was significantly high among married people, among students and among those people doing jobs in different offices as profession. In this study majority of the participants were male who are highly educated (62\% having graduate level education). For the stress subscale, out of 510 participants $189(51.4 \%)$ male and $63(44 \%)$ female were considered to have normal score $(0-10)$.Mild to moderate score $(11-26)$ was of $112(30.5 \%)$ male and 42(29.3\%) female. Severe to extremely severe score (27-42) was found among 66(18\%) male and 38(1926.5\%) were female Respondents. Depression was also significantly high among student and people doing jobs in Government, semi Government and Private offices. Depression in psychology is a mood or emotional state marked by feelings of low self-worth or guilt and a reduced ability to enjoy life. For depression subscale, $152(41.4 \%)$ male and $56(39 \%)$ female were considered to have normal score (0-9). Mild to moderate score (10-20) was of $110(30 \%)$ male and 42 (29\%) were female participants. Severe to extremely severe score (21-42) was found among 105(29\%) male and 45(31.4\%) were female respondents. Whereas, Anxiety is an emotion characterized by feelings of tension, worried thoughts and physical changes like increased blood pressure. For anxiety subscale, $142(39 \%)$ male and $58(40 \%)$ female were considered to have normal score (0-7). Mild to moderate score (8-14) was of 101(28\%) male and $37(26 \%)$ were female. Severe to extremely severe score (15-42) was found among 124(34\%) male and $48(33 \%)$ were female participants. There is no significance of anxiety as marital status and education wise. Only the students are anxious about their studies and future.

The study found that the most common reason for stress and anxiety among general population due to COVID-19 was the Possibility of expanding COVID-19 duration (81.4\%) (Table - 3), fear about their family members $(52.2 \%)$, followed by the fear of getting infected themselves $(28.0 \%)$. Other reasons were: job insecurity, lack of treatment facilities and lack of awareness among the general population about COVID-19. In this study, majority of the participants show that during the initial stage of COVID-19 epidemic, internet (48.2\%) was chief and primary source of health information for the general population. Nearly all of the participants updated by the latest information as rout of transmission, efficiency and availability of medicines, advice on prevention, location and area-wise number of infected cases, number of death and number of recovered cases. Results of this study show that up to date information about corona recovered cases help to decrease the level of stress in population. The results also displays that most of the participants (75.5\%) were satisfied with the extent of available health information. Higher the satisfaction with available health information, associate with lower psychological effects, depression, anxiety, stress and fear from outbreak. Regarding the precautionary measures advice by TV and other source to prevent from this COVID infection, more than half of the respondents washed their hands with soaps after coming from outside the home and after using the outside item, always using a mask in the presence or absence of virus infection / symptoms, most of the participants were already well mannered and always covering mouth before coughing and sneezing and about half of the participants always avoiding the sharing utensils (e.g., spoon, fork, glass and tea cups) during the meal. The collected data of the survey present that women were at high level of depression and stress due to spreading of this infection as most of the women of our country are housewives and manage financial and social matter of their family. These results are similar to other research of psychological health information presented that women of any society are always found in depress condition [10]. Due to increasing number of patient of corona virus disease in major cities of Pakistan health authority announced to close all educational institutes (school, college, universities and deeni madaris) to overcome the risk of virus transmission by face to face therapy but psychologically students and their parents were very depressed as they were very anxious about their future. Owing to negative impact on academic progression, education authorities requested to manage online portals and web-based service-for teaching activities [11]. Todays, children and young population are more interested to use smartphone applications in any society [12], health authorities provide online psycho-education and psychological intervention to reduce the risk of corona virus spreading. Online education system is also useful and offer provision for those people who are not educated and spent most of the time in home due to virus pandemic. Our survey research used as historical reference and provides baseline data for minimizing the psychological effects during COVID infection period.

\section{Conclusions}

As the COVID infection spreading continuously, this study is an effort to provide aspirated guidance for psychological patients and the persons who are in stress and depression due to corona virus outbreak.

Result show that most of the participants are aware about the spreading factor of this infection but worried about the fear of getting infected themselves and family members. High levels of anxiety, stress and depression among general population were noted, which is a cause for concern. Both the government and health care agencies are responsible for protecting the psychological well-being all over the world and ensuring a healthy work environment. As the second wave of pandemic spreading rapidly, it is necessary to take serious steps and must prepare preventive health care systems and need a high level of action, planes and management at national level.

This publication is licensed under Creative Commons Attribution CC BY. 


\section{References}

[1]- Mukhtar S. Preparedness and proactive infection control measures of Pakistan during COVID-19 pandemic outbreak.Res Social Adm Pharm 2020; S15517411(20)30373-9.

[2]- Wang C, Pan R, Wan X, Tan Y, Xu L, Ho CS, et al. Immediate psychological responses and associated factors during the initial stage of the 2019 coronavirus disease (COVID-19) epidemic among the general population in China. Int J Environ Res Public Health2020; 17(5):1729.

[3]- Chen, N. Epidemiological and clinical characteristics of 99 cases of 2019 novel coronavirus pneumonia in Wuhan, China: A descriptive study. Lancet 2020, 395, 507-513. [CrossRef]

[4]- Holshue, M.L. First Case of 2019 Novel Coronavirus in the United States. N. Engl. J. Med. 2020. [CrossRef][PubMed]

[5]- Nishiura, H. The Rate of Underascertainment of Novel Coronavirus (2019-nCoV) Infection: Estimation Using Japanese Passengers Data on Evacuation Flights. J. Clin. Med. 2020, 9, 419. [CrossRef]

[6]- Lovibond SH, Lovibond PF:Manual for the Depression Anxiety Stress Scales. 2nd edition. Sydney: Psychology Foundation of Australia; 1995.[9].

[7]- Ho, C.S.H. Relationship of Anxiety and Depression with Respiratory Symptoms: Comparison between Depressed and Non-Depressed Smokers in Singapore. Int. J. Environ. Res. Public Health 2019, 16, 163.[CrossRef]

[8]- Quek, T.C. Misophonia in Singaporean Psychiatric Patients: A Cross-Sectional Study. Int. J. Environ. Res. Public Health 2018, 15, 1410. [CrossRef]

[9]- McAlonan, G.M. Immediate and sustained psychological impact of an emerging infectious disease outbreak on health care workers. Canadian journal of psychiatry. Revue Canadienne De Psychiatrie 2007, 52, 241-247.[PubMed]

[10]- Lim, G.Y. Prevalence of Depression in the Community from 30 Countries between 1994 and 2014. Sci. Rep. 2018, 8, 2861. [CrossRef] [PubMed]

[11]- Zhang, M.W.B.; Ho, C.S.H.; Ho, R.C.M. Methodology of development and students' perceptions of a psychiatry educational smartphone application. Technol. Health Care O

. J. Eur. Soc. Eng. Med. 2014, 22, 847-855. [CrossRef]

[12]- Do, T.T.T. Receptiveness and preferences of health-related smartphone applications among Vietnamese youth and young adults. BMC Public Health 2018, 18, 764. [CrossRef] 